\title{
Ser joven estudiante y no morir en el intento
}

\section{Being a young student and not to die trying}

\author{
Carlota Guzmán Gómez y Claudia Saucedo Ramos (coords.) \\ (2007), LA VOZ DE LOS ESTUDIANTES. EXPERIENCIAS EN TORNO A LA ESCUELA, \\ Universidad Nacional Autónoma de México-Ediciones Pomares, \\ MÉXICO, 222 PP., ISBN: 978-84-8782-84-7
}

El joven de hoy creció creyendo que en el siglo xxi la tecnología haría que su vida fuera mucho más sencilla y que tendría muchas más posibilidades de ser exitoso, en comparación con sus padres; también se le enseñó que la educación formal -vía la escuela- sería el elemento disparador de una mejor calidad de vida. Lo que no se le explicó es que su generación (jóvenes de entre 15 y 24 años de edad) es muy voluminosa (en 2005 representó la quinta parte de la población mexicana), tampoco le dijeron que dada la inercia demográfica, durante algunos años más ejercerá junto con toda su generación una fuerte presión para acceder al sistema escolar (medio superior y superior), ${ }^{1}$ y desde luego para competir en los limitados y precarios puestos laborales. ${ }^{2}$

En el libro La voz de los estudiantes las coordinadoras, y autoras de dos artículos, Carlota Guzmán ${ }^{3}$ y Claudia Saucedo ${ }^{4}$ reúnen ocho trabajos en los que utilizan el enfoque cualitativo a través de entrevistas a profundidad, de narraciones elaboradas por los mismos estudiantes, mediante cuestionarios y vía una profunda observación, nos llevan a conocer qué opinan los estudiantes, cuáles son los procesos subjetivos que viven y dan sentido a su paso por diversas instituciones del nivel medio superior y superior. ${ }^{5}$ En este libro se señalan y analizan muchas experiencias estudiantiles, múltiples trayectorias y también la diversidad que gira en torno a los jóvenes bachilleres mexicanos.

${ }^{1}$ En 2005, en el primer proceso de selección que llevó a cabo la UNAM, fueron aceptados 9,749 estudiantes de un total de 100,900 aspirantes; el mismo año, en la UAM hubo 35,000 aspirantes y fueron aceptados 7,000 alumnos (citado en ANUIEs, 2007).

${ }^{2}$ En 2003, del total de la población económicamente activa (PEA), el grupo de edad 20 a 24 años tuvo el mayor porcentaje de desempleados (poco más de 25\%), entre el grupo de 15 a 19 la cifra fue de alrededor de $20 \%$. Poco más de la mitad de los jóvenes asalariados no contaron en el mismo año con seguridad social; y en relación con el ingreso obtenido, resalta que los jóvenes recibieron por hora trabajada 30\% menos que lo que percibió un adulto (Pacheco, 2008).

${ }^{3}$ Doctora en ciencias de la educación por la Universidad de París VIII, investigadora del Centro Regional de Investigaciones Multidisciplinarias de la UnAM.

${ }^{4}$ Doctora en investigaciones educativas por el DIE-CINVESTAV-IPN, investigadora en la Facultad de Estudios Superiores de la unAM, en la carrera de psicología.

${ }^{5}$ De los ocho trabajos, un texto remite a estudiantes del nivel superior. 
Con diferentes miradas y referentes teóricos que van de las ciencias sociales a la psicología, rompiendo las fronteras disciplinares, se nos abre una gran ventana para conocer desde la lógica del estudiante el valor e importancia que ellos y ellas dan a la escuela, no sólo como espacio formativo, sino como espacio cultural, espacio de vida. Gran acierto.

Leer un libro en el que se conozca el panorama de los estudiantes no es un tema nuevo, desde hace décadas se ha abordado pero, la mayoría de las veces, se ha hecho desde una perspectiva estadística: cuántos son, en qué nivel educativo se encuentran, su situación según el género, han sido temas tratados y claves fundamentales para la política educativa. Pero ver al estudiante como sujeto de la educación, conocer sus experiencias en la escuela sí es un tema novedoso y es lo que resalta en este texto.

La voz de los estudiantes. Experiencias en torno a la escuela busca entender los significados que estudiantes del nivel medio superior del Distrito Federal y del Estado de México le dan a la escuela, esto se analiza a partir de sus distintos contextos familiares e individuales, se analiza a partir del tipo de espacio educativo al que asisten (de modalidad tecnológica o universitaria; instituciones que consideran -desde su opinión- buenas o malas, es decir, que cumplen o no con sus expectativas). Sus distintas realidades hacen que ellos y ellas construyan una serie de significados y actitudes hacia la escuela en función de cómo la viven cada día, pero también de cómo suponen son percibidos por los otros (maestros y estudiantes).

El trabajo se divide en cuatro apartados que muestran: las trayectorias educativas, los valores y revaloraciones en torno a la escuela, los discursos que se construyen a partir de la percepción que se tiene de la escuela y la búsqueda de la identidad, todo analizado desde la mirada de los estudiantes.

Se comienza por señalar la diversidad de las trayectorias educativas de los y las estudiantes, y se ejemplifica cómo el pertenecer al mismo grupo de edad o contar con condiciones socioeconómicas similares no necesariamente obliga a darle valor igual a la escuela. Las necesidades y motivaciones que permiten o dificultan terminar un nivel escolar son muy amplias, y entre los jóvenes se construyen a cada momento, sin sentido prefigurado del todo ni con un punto de arribo único. Los y las jóvenes cada día ponen a prueba su vínculo y su permanencia en la escuela en función de la historia familiar y de la historia individual. Queda claro que en el terreno educativo no hay una trayectoria única o sin rupturas, de hecho, los menos son quienes cumplen con la trayectoria ideal.

En relación con las valoraciones y revaloraciones en torno al sentido de la escuela, los dos autores que llevan a cabo este tema identifican los distintos significados que tiene la escuela para los alumnos de bachillerato (uno de modalidad técnica y otro universitaria). Se establecen las di- 
ferencias entre los alumnos en función del tipo de bachillerato al cual asisten y se concluye que ambas modalidades educativas, más que promover una igualdad entre los jóvenes, está generando condiciones de exclusión para los que provienen de la modalidad tecnológica.

Cómo perciben los estudiantes la llegada a una institución educativa que no es de su agrado, que no fue de su elección y cómo viven el ejercicio del poder en las instituciones de nivel medio superior (en especial se analiza el caso de un Centro de Estudios Tecnológicos) se discute en el tercer apartado. Estudiar en una institución que no es del gusto de los jóvenes se traduce en vivir la escuela con sufrimiento, con la sensación de rechazado, lo que conlleva de inicio un sentido de devaluación de sí mismo; y por otra parte estudiar en un ámbito que se percibe como de doble moral y donde no se permite ni se dan las herramientas para actuar de manera independiente o autónoma, repercute en el desarrollo sociomoral del joven estudiante.

El cuarto y último bloque tiene que ver con la búsqueda de la identidad de los estudiantes. En el primer trabajo de esta sección, a partir de autobiografías, se buscó conocer cuáles son las condiciones de desarrollo psicosocial que tienen los jóvenes bachilleres, logrando identificar diferentes posiciones en el camino a la conquista de la identidad, que van desde una identidad negativa, la confusión, hasta la identidad conquistada.

El último trabajo no tiene que ver con jóvenes bachilleres sino con estudiantes universitarios y no menciona solamente el aspecto educativo, sino entra en juego el tema laboral, que se consideraría el escalón siguiente a la conclusión del nivel superior. En este texto se elaboran cuatro categorías identitarias, a saber: estudiantes, estudiantes trabajadores, trabajadores estudiantes e individuos, categorías que tienen que ver con las múltiples experiencias y actividades que viven los jóvenes y que se trastocan al alternar el estudio y el trabajo. La autora sostiene que si bien a este conjunto de jóvenes los unifica el ser estudiantes y trabajadores, este hecho no los convierte en un grupo homogéneo, pues la forma de vivir ambas actividades de manera simultánea tiene que ver con sus condiciones e intereses personales y con los motivos que encuentran en su quehacer laboral.

El libro, en definitiva, nos muestra un vasto panorama de la percepción y significado que tienen los estudiantes bachilleres sobre la escuela, pero, desde mi punto de vista, maneja varios temas nodales.

1) Evidencia la heterogeneidad de este grupo al que lo único que lo une es su pertenencia a un nivel educativo.

2) Se señala este imaginario compartido de que sólo la educación preparatoria, con miras a alcanzar el nivel superior vía la universidad tiene validez. Las opciones técnicas representan un descalabro 
en la vida de los jóvenes pues dificultará su acceso a estudios universitarios. El ser licenciado sigue dando un estatus de éxito que los jóvenes buscan.

3) Otro punto que se vislumbra en la lectura es que la sociedad cambia, pero tal parece que las escuelas no. Pareciera que el sistema educativo no se ha percatado de que si bien antes las exigencias básicas tenían que ver con alfabetizar y dar una socialización bási$\mathrm{ca}$, ahora se deben comunicar competencias y conocimientos relevantes para responder a nuevas exigencias culturales y laborales para estos ciudadanos exigentes que son los jóvenes. Esto es más evidente cuando ellos y ellas expresan su opinión acerca de los centros que imparten enseñanza técnica.

En conclusión, La voz de los estudiantes. Experiencias en torno a la escuela cumple su objetivo al dar voz a quienes no la tienen: los jóvenes estudiantes. Pero no hay que dejar de anotar que si bien en algunos artículos se presenta a un grupo de jóvenes vulnerable y con problemas, este grupo sigue siendo privilegiado, por ejemplo, según el Conteo de Población de 2005, sólo $47.8 \%$ de la población de 15 a 19 años, ${ }^{6}$ asistía a la escuela; cifra que resulta muy pequeña sobre todo porque se sigue considerando que ser joven es sinónimo de ser estudiante.

Aunque parece retórica, pensar en los jóvenes de hoy, en los jóvenes estudiantes, no es sólo pensar en el presente sino es hacerlo en el futuro. Los jóvenes estudiantes de hoy conforman la población que permitirá el crecimiento del país en los años por venir y de no invertir en ellos, de no resolver los problemas y demandas reales de acceso a la educación y de sus oportunidades de empleo, el potencial demográfico que representan estará perdido.

\section{Bibliografía}

ANUIES (Asociación Nacional de Universidades e Instituciones de Educación Superior) (2007), Retención y deserción en un grupo de instituciones mexicanas de educación superior, ANUIEs, México.

Pacheco, Edith (2008), "Los jóvenes y el trabajo en México”, en Fortino Vela (coord.), La dinámica demográfica y su impacto en el mercado laboral de los jóvenes, México, Universidad Autónoma Metropolitana, Xochimilco, México, pp. 135-158.

${ }^{6}$ De acuerdo con esta fuente, en el Distrito Federal la cifra ascendió a 63\%, pero en el Estado de México fue de $49.5 \%$. 
Recibida: 19 de marzo de 2010. Aceptada: 7 de abril de 2010.

Emma Liliana Navarrete El Colegio Mexiquense

Correo e: enavarr@cmq.edu.mx

Emma Liliana Navarrete. Es antropóloga social por la Universidad Autónoma Metropolitana, Iztapalapa, maestra en demografía por El Colegio de México y doctora en estudios de población también por El Colegio de México. Actualmente es profesora-investigadora en El Colegio Mexiquense. Sus principales áreas de investigación son los jóvenes, el trabajo y el entorno laboral de mujeres universitarias. Sus publicaciones más recientes son: "Nivel de escolaridad y oportunidades laborales para las mujeres mexiquenses al inicio del siglo XxI", en Emma Liliana Navarrete (coord.), $\mathrm{Mu}$ jeres mexiquenses. Pasado y presente de las voluntades que transforman, Biblioteca Mexiquense del Bicentenario, Serie Nuevo Pensamiento, pp. 237-261 (2009); "Trabajo y universitarias. Mujeres mexiquense de dos generaciones", en Luz María Salazar (coord.), Sujetos laborales del Estado de México, El Colegio Mexiquense, Toluca, pp. 153-175 (2009). 